\title{
New perspectives for multi-level regulations of neuronal acetylcholinesterase by dioxins
}

\author{
Heidi Q. Xie ${ }^{\text {a, b, } 1}$, Tuan Xu ${ }^{\text {a, b, }}{ }^{\text {, }}$, Yangsheng Chen ${ }^{\text {a, b }}$, Yunping Li ${ }^{\text {a, b }}$, Yingjie Xia ${ }^{\text {a, b }}$, \\ Sherry L. Xu ${ }^{\text {a, b }}$, Lingyun Wang ${ }^{\text {a, b }}$, Karl W.K. Tsim ${ }^{c}$, Bin Zhao ${ }^{\text {a, b, * }}$ \\ a State Key Laboratory of Environmental Chemistry and Ecotoxicology, Research Center for Eco-Environmental Sciences, Chinese Academy of Sciences, \\ Beijing, China \\ ${ }^{\mathrm{b}}$ University of Chinese Academy of Sciences, Beijing, China \\ ${ }^{c}$ Division of Life Science, Center for Chinese Medicine and State Key Laboratory of Molecular Neuroscience, The Hong Kong University of Science and \\ Technology, Clear Water Bay Road, Hong Kong, China
}

\section{A R T I C L E I N F O}

\section{Article history:}

Received 8 January 2016

Received in revised form

22 April 2016

Accepted 29 June 2016

Available online 1 July 2016

\section{Keywords:}

Dioxins

Acetylcholinesterase

MicroRNA

Transcription

Signaling pathway

\begin{abstract}
A B S T R A C $T$
Acetylcholinesterase (AChE; EC 3.1.1.7) is a vital functional enzyme in cholinergic neurotransmission which can rapidly hydrolyze neurotransmitter, acetylcholine, in the central and peripheral nervous systems. Emerging evidence showed that in addition to classical environmental AChE inhibitors, e.g. organophosphate and carbamate pesticides, dioxins are a new type of xenobiotic causing impairment of AChE. Dioxin can transcriptionally or post-transcriptionally suppress AChE expression in human neuroblastoma cells or mouse immune cells via the aryl hydrocarbon receptor (AhR) pathway, respectively. Dioxins can affect gene expression through other mechanisms, such as cross-talk with other signaling cascades and epigenetic modulations. Therefore, in this review, by summarizing the known mechanisms of AChE regulation and dioxin-induced gene alteration, potential signaling cascades and epigenetic mechanisms are proposed for dioxin-mediated AChE regulation. Mitogen activated protein (MAP) kinase, $3^{\prime}, 5^{\prime}$-cyclic adenosine monophosphate (cAMP) and calcium-related singaling pathways, as well as potential epigenetic mechanisms, such as DNA methylation, and post-transcriptional regulation via microRNAs, including hsa-miR-132, hsa-miR-212 and hsa-miR-25-3p are discussed here. These proposed mechanisms may be invaluable not only to promote comprehensive understanding of the action mechanisms for dioxin, but to illustrate the molecular basis of dioxin-induced health impacts.
\end{abstract}

๑) 2016 Elsevier Ireland Ltd. All rights reserved.

\section{Introduction}

Acetylcholinesterase (AChE; EC 3.1.1.7) plays a key role in cholinergic synapses in central and peripheral nervous systems by rapid hydrolyzing the neurotransmitter, acetylcholine [1]. It has been known for a long time that organophosphate and carbamate pesticides are able to specifically inhibit the enzymatic activity of AChE in different species. In particular, high level of exposure to the pesticides leads to acute neurotoxicity in humans [2]. In recent

Abbreviations: AChE, acetylcholinesterase; AhR, aryl hydrocarbon receptor; MAP, mitogen activated protein; cAMP, 3', 5'-cyclic adenosine monophosphate; miRs, microRNAs.

* Corresponding author. Research Center for Eco-Environmental Sciences (RCEES), Chinese Academy of Sciences, Beijing, 100085, China.

E-mail address: binzhao@rcees.ac.cn (B. Zhao).

1 These authors contributed equally to this study. years, various environmental chemicals are found to impair AChE function, such as persistent organic pollutants which are drawing particular attention recently. These chemicals are persistent in the environment, accumulate in the biota through the food chain, and exert ecological toxicities and health impacts even after being eliminated from the environment [3]. Dioxins are representative of such chemicals whose toxicity and action mechanisms have been extensively investigated [4]. Dioxins, including polychlorinated dibenzodioxins and dibenzofurans, as well as polybrominated dibenzo-p-dioxins and dibenzofurans, are unintentionally produced by industrial processes and incinerations [4]. They exhibit a wide range of toxic effects (e.g., liver toxicity, immune system impairment, developmental deficit in the nervous system etc.) [5]. Gestational or adult exposure of dioxin was associated with a loss of higher-order brain functions (e.g., emotion, cognition and psychological disorders) in animals and humans [6]. It is generally accepted that dioxins exert their biological and toxicological effects 
by transcriptional gene regulation via the aryl hydrocarbon receptor (AhR) pathway [6]. In addition, the cross-talk of AhR pathway with various intracellular signaling cascades was proposed to be involved in the toxicity triggered by dioxins [7].

Interference of dioxin and dioxin-like compounds with AChE and other cholinergic molecules were first reported as indirect effects due to hypothyroidism-induced developmental deficits in the nervous system [8]. Upon maternal administration of dioxin, the brain tissue of the offspring exhibited lower enzymatic activity of AChE compared to control rats [8]. Dioxin-induced hypothyroidism was proposed as the mechanism for the developmental defect of AChE [8]. This indirect effect was consistent with that of Aroclor 1254 (a mixture of dioxin-like and non-dioxin like polychlorinated biphenyl (PCB)), in which choline acetyltransferase (ChAT) activity was reduced [9]. Studies by us and others have demonstrated that dioxins suppress AChE expression directly in neuroblastoma cells and immune cells upon dioxin exposure, in which transcriptional regulation via $A h R$ and post-translational regulation via microRNA (miR) were involved, respectively [10-12]. Since the dioxininduced gene alterations involve a complex multi-level regulatory network, present knowledge on how dioxins affect AChE is not sufficient. Therefore, we have summarized intersectional mechanisms for AChE and dioxin-related gene regulation at the transcriptional and post-transcriptional levels, as to provide hints to uncover a full picture of the underlining mechanisms for dioxin effects.

\section{Potential signaling cascades beyond AhR-dependent pathways}

Our previous work demonstrated that dioxin decreased AChE activity by transcriptional suppression of the $\mathrm{AChE}_{\mathrm{T}}$ subunit, a major variant of AChE transcript in the nervous system, in SK-N-SH human neuroblastoma cells: this suppression was mediated via AhR-dependent pathway [10]. This transcriptional alteration of AChE led to a decrease in the protein expression, which consequently caused the decrease in AChE enzymatic activity[10]. There was no effect on the $A C h E_{R}$ subunit, a rare splicing variant in the brain, nor on proline-rich membrane anchor (PRiMA), a structural subunit of neuronal AChE [10]. Consensus sequences of dioxin responsive element were found in the regulatory region upstream of the human ACHE gene, which might mediate the above transcriptional regulation [10]. Whether dioxin could influence AChE expression via acting on known AChE-related signaling pathways in muscle and neurons needs further investigation.

Mitogen activated protein (MAP) kinase has been demonstrated to be involved in the transcriptional up-regulation of $\mathrm{AChE}_{\mathrm{T}}$ and its structural subunit, PRiMA, during neuronal differentiation [13,14]. A recent study on neuro-beneficial effects of phyto-chemicals also suggested that the MAP kinase pathway could mediate xenobioticinduced alterations of AChE in PC-12 cells [15,16]. Interestingly, the MAP kinase pathway also participated in the toxicological effects of dioxin. In cultured cerebellar granule cells, the MAP kinase was involved in dioxin-induced reactive oxygen species production [17]. Moreover, this pathway also had cross-talk with AhR and participated in dioxin effects in cancer and dendritic cells, as well as during osteoblast differentiation [18-20]. These pieces of evidence suggest a possible involvement of the MAP kinase pathway in AChE regulation triggered by dioxin.

As one of the secondary messengers, the $3^{\prime}, 5^{\prime}$-cyclic adenosine monophosphate (CAMP) signaling was proposed to be involved in the tight control of AChE and collagen Q (ColQ) expression, during myogenesis and muscle innervation [21-25]. Accordingly, enhancement of cAMP-signaling by administration of db-cAMP or foskolin was found to interfere with dioxin-dependent induction of
CYP1A1 in mouse hepatoma cells [26]. On the other hand, the inhibitory effect of dioxin on cAMP-induced elevation of a glial differentiation marker, GFAP, was documented in cultured C6 glial cells [27]. Although there is no solid evidence showing a direct interaction of cAMP signaling and AhR signaling in neurons, the evidence of having cross-talk between these two signalings raises a possible involvement of cAMP in dioxin effects on neuronal AChE expression.

Apart from these two signaling cascades, calcium is another possible signaling molecule that is involved in dioxin-mediated AChE modulation. Dioxin was found to induce apoptosis by disruption of intracellular calcium homeostasis in human neuronal cell line SHSY5Y [28]. On the other hand, a signaling cascade triggered by calcium mobilization was involved in P2Y2 receptorinduced AChE expression in rat cortical neurons [14]. Thus, calcium-related signaling cascade(s) might be a candidate responsible for dioxin-induced transcriptional alterations of AChE.

\section{Epigenetic regulatory mechanisms}

\subsection{Potential roles of DNA methylation and histone modification}

Transcriptional activity of a gene can be regulated by epigenetic processes, involving DNA methylation and histone modification and microRNAs(miRs) expression [29]. Lau et al. demonstrated that after administration of a DNA methyltransferase inhibitor, the expression profile of AChE changed dramatically during the myogenesis process in $\mathrm{C} 2 \mathrm{C} 12$ cells [30]. One SP1 site located $1826 \mathrm{bp}$ upstream of the mouse $A C H E$ gene was revealed to be methylated in this study [30]. Sailaja et al. investigated the epigenetic mechanism of the stress-induced long-lasting decrease of two AChE splicing variants distinct at the $5^{\prime}$ exons in hippocampus. Alteration of the splicing variants was due to reduced acetylation and elevated trimethylation of H3K9 at the corresponding promoter, in which histone deacetylase 4 served as a mediator [31].

Accordingly, emerging evidence has shown involvement of dioxins in epigenetic processes. Dioxin exposure has been associated with changes in DNA methylation status, that might in turn affect the dioxin-induced gene expression in splenocytes [32]. In addition to experimental data, high serum levels of dioxin OCDD and one dioxin-like PCB, PCB126, were associated with global DNA hypermethylation in an elderly population [33].

Thus the interference of dioxins with global DNA methylation might result in general alterations of a series of genes, perhaps including AChE. It is possible that altered DNA methylation levels induced by dioxin or dioxin-like compounds might particularly affect transcriptional properties of AChE in brain and/or in muscle.

\subsection{Potential post-transcriptional modulations via dioxin-related miRs}

MicroRNAs (miRs) are defined as short noncoding RNA molecules, 18-25 nucleotides in length, and their main function is to post-transcriptionally down-regulate expression of their target genes by inhibiting the translation or inducing the mRNA degradation. In the nervous system, miRs have been definitively linked to development, synaptic plasticity and neurodegenerative diseases [34]. Neuronal AChE is also regulated by miRs. In murine cell lines, miR-132 has been documented to target $\mathrm{AChE}_{\mathrm{T}}$ mRNA and consequently affect the AChE expression during stress-induced cognitive impairment [35]. In addition, primate-specific miR-608 was recently found to target $\mathrm{AChE}$ in human-derived cell lines [36]. By using PicTar, miRanda, miRbase, and microCosm algorithms, 47 miRs were predicted to target the human $\mathrm{AChE}_{\mathrm{T}}$ variant, and 81 miRs to target human $\mathrm{AChE}_{\mathrm{R}}$ [37]. By integrating the predicted data 
Table 1

A list of dioxin-induced miRs in human cell lines.

\begin{tabular}{|c|c|c|c|c|}
\hline miRBase: Accession & $\operatorname{miR}$ & Regulation & Cell/tissue type & Reference \\
\hline MIMAT0000440 & hsa-miR-191-5p & up & HepG2 cells & [39] \\
\hline MIMAT0000765 & hsa-miR-335-5p & up & MDA-MB-231 and BT474 cells & [40] \\
\hline MIMAT0000269 & hsa-miR-212-3p & up & MDA-MB-231 and T47D cells & [41] \\
\hline MIMAT0000426 & hsa-miR-132-3p & up & MDA-MB-231 and T47D cells & \\
\hline MIMAT0000081 & hsa-miR-25-3p & up & Multiple myeloma cells & {$[42]$} \\
\hline MIMAT0000092 & hsa-mir-92a-3p & up & Multiple myeloma cells & \\
\hline
\end{tabular}

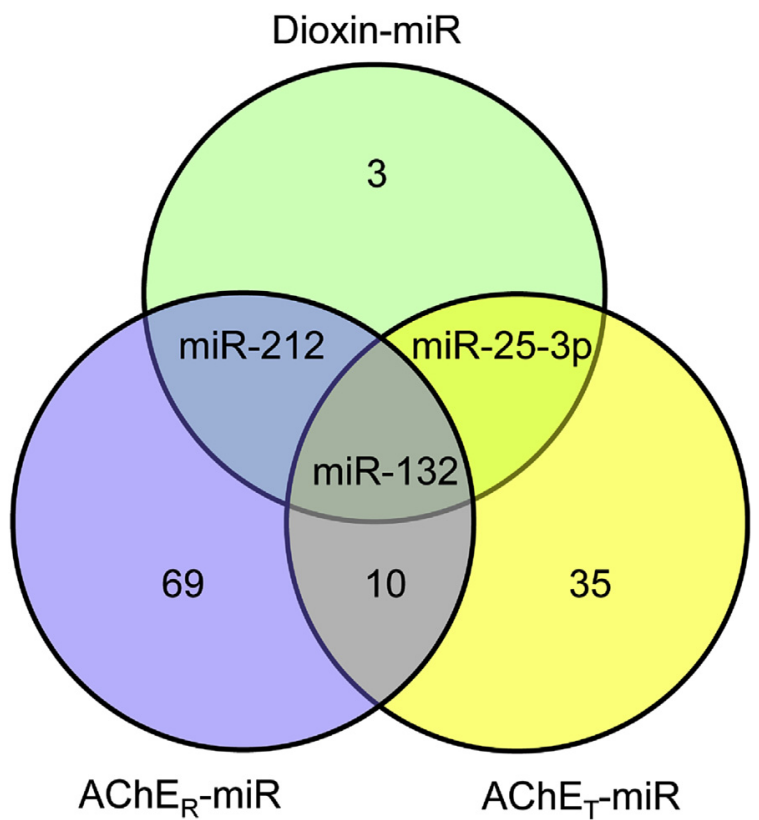

Fig. 1. Potential miRs mediateing post-transcriptional regulation of AChE by dioxins. A three-way Venn diagram is shown. The lower circle at left hand side represents $A C h E_{R^{-}}$ targeting miRs $\left(A C h E_{R}-m i R\right)$, the one on the right represents $A C h E_{T}$-targeting miRs $\left(A C h E_{T}-m i R\right)$ and the circle on the top represents the reported miRs induced by dioxin (Dioxin-miR). The numbers express the number of miRs present in each independent of overlapping sub-group. The names of intersecting miRs between either group of AChE miRs and the dioxin-miR group, and among the three groups of miRs are given. These are potential candidate regulatory miRs. from this study with dioxin-related miRs, we could find possible candidates of miRs involving in dioxin-induced AChE alterations.

It was demonstrated that dioxin exposure triggered the upregulation of mmu-miR-132 via AhR, which caused downregulation of $\mathrm{AChE}$ in $\mathrm{CD}^{+} \mathrm{T}$ cells and attenuation of experimental autoimmune encephalomyelitis [12]. This is the first evidence showing miRs mediating a dioxin effect on AChE expression. miR-132 was also involved in the promotion of synaptogenesis in rat hippocampal neurons by a non-dioxin-like PCB, PCB-95 [38]. In addition to miR-132, accumulating evidence showed that dioxins could interfere with biogenesis and expression of various miRs in different human-derived cells (Table 1). Six different miRs, including hsa-miR-191, hsa-miR-335, hsa-miR-212, hsa-miR-132, hsa-miR-25-3p and hsa-mir-92a-3p, were all significantly upregulated by dioxin via AhR signaling pathway. These miRs altered by dioxin occurred in other non-neuronal tissues.

By finding the overlapping miRs from the aforementioned prediction, more candidate miRs mediating dioxin effects on AChE may be identified. Fig. 1 shows a Venn plot demonstrating the intersection of the three groups of miRs, including $A C h E_{\mathrm{T}}$-targeting miRs $\left(A C h E_{T}-m i R\right), A C h E_{R}$-targeting miRs $\left(A C h E_{R}-m i R\right)$ and miRs altered by dioxin. In line with the literature, hsa-miR-132 is present in all groups and thus has the highest potential for mediating dioxin effects on AChE. Hsa-miR-212 is present at the intersection of the $\mathrm{AChE}_{\mathrm{R}}-\mathrm{miR}$ and dioxin-miRs groups, while hsa-miR-25-3p is at the intersection of the $\mathrm{AChE}_{\mathrm{T}}-\mathrm{miR}$ and dioxin-miRs groups. Thus the latter two candidate miRs also have potential for being involved in post-transcriptional regulation of $\mathrm{AChE}$ by dioxins. However, tissue-specific effects of the miRs should be considered in further investigations.

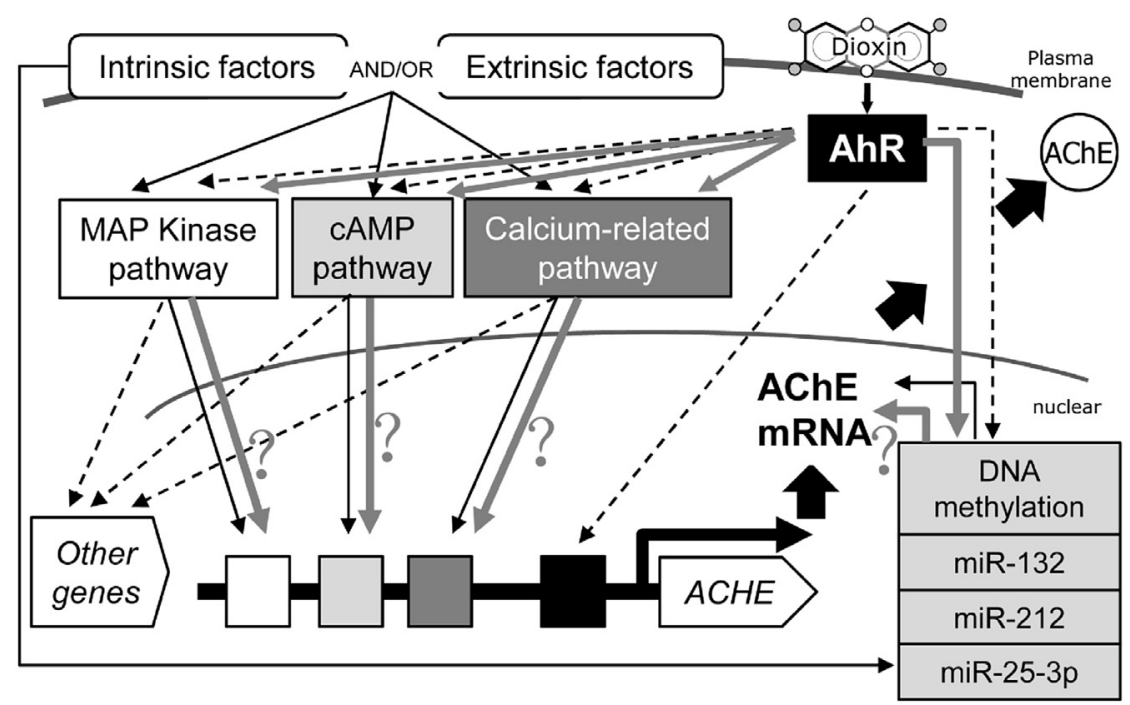

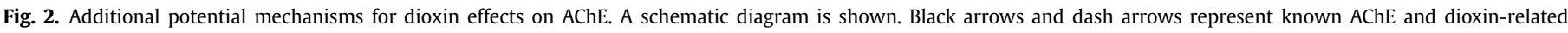
regulatory mechanisms, respectively. Grey arrows and question marks stand for the additional potential mechanisms for dioxin effects on AChE. 


\section{Summary}

As an important enzyme in cholinergic neurotransmission, the expression of $\mathrm{AChE}$ is tightly controlled via multi-level regulatory mechanisms including transcriptional mechanisms involving various signaling cascades, epigenetic regulation to preserve longterm transcriptional status and post-transcriptional control of mRNA levels via miRs. Disturbances and alterations of any combination of these mechanisms may occur during toxicological or pathological processes resulting from exposure to toxic xenobiotics or pathogens. These abnormal processes may lead to altered expression and enzymatic activity of AChE, and subsequent malfunction of the cholinergic nervous system.

Here, we focused on a new type of highly toxic xenobiotics, dioxins, which were recently found to alter AChE expression in human neuroblastoma cells and mouse immune cells. The documented action mechanisms for the effects are limited to AhRdependent transcriptional and post-transcriptional regulation via miR-132. After surveying the literature documenting dioxin effects in different cell types and tissues, we have proposed several additional potential mechanisms for dioxin effects on AChE (Fig. 2). The candidate mechanisms include MAP kinase, cAMP-dependent and calcium-dependent signaling cascades and potential epigenetic mechanisms, such as DNA methylation and post-transcriptional regulation via hsa-miR-132, hsa-miR-212 or hsa-miR-25-3p. Most of the proposed mechanisms are involved in stress or pathology of certain diseases, such as neurodegenerative diseases. Therefore, further study of the proposed mechanisms might also help to illustrate the molecular linkage of dioxin exposure with health impacts.

\section{Acknowledgements}

This work was supported by the National Natural Science Foundation of China (No. 21177150, 21377160, 21525730), the Strategic Priority Research Program of the Chinese Academy of Sciences (No. XDB14030401, XDB14030402), and we thank Dr. Marjorie A. Phillips from UC Davis for comments during the manuscript preparation.

\section{Transparency document}

Transparency document related to this article can be found online at http://dx.doi.org/10.1016/j.cbi.2016.06.030.

\section{References}

[1] J. Massoulié, The origin of the molecular diversity and functional anchoring of cholinesterases, Neurosignals 11 (3) (2002) 130-143.

[2] M.G. Lionetto, et al., Acetylcholinesterase as a biomarker in environmental and occupational medicine: new insights and future perspectives, Biomed. Res. Int. 2013 (2013) 321213.

[3] J.R. Suarez-Lopez, et al., Persistent organic pollutants in young adults and changes in glucose related metabolism over a 23-year follow-up, Environ. Res. 137 (2015) 485-494.

[4] S. Li, et al., Comparison of the contributions of polychlorinated dibenzo-pdioxins and dibenzofurans and other unintentionally produced persistent organic pollutants to the total toxic equivalents in air of steel plant areas, Chemosphere 126 (2015) 73-77.

[5] R.A. Budinsky, et al., Mode of action and dose-response framework analysis for receptor-mediated toxicity: the aryl hydrocarbon receptor as a case study, Crit. Rev. Toxicol. 44 (1) (2014) 83-119.

[6] H.M. Korashy, H.M. Abuohashish, Z.H. Maayah, The role of aryl hydrocarbon receptor-regulated cytochrome P450 enzymes in glioma, Curr. Pharm. Des. 19 (40) (2013) 7155-7166.

[7] T.-H. Hsieh, et al., Phthalates induce proliferation and invasiveness of estrogen receptor-negative breast cancer through the AhR/HDAC6/c-Myc signaling pathway, FASEB J. 26 (2) (2012) 778-787.

[8] R.G. Ahmed, Perinatal TCDD exposure alters developmental neuroendocrine system, Food Chem. Toxicol. 49 (6) (2011) 1276-1284.
[9] L.M.J. de Ku, et al., Thyroxine normalizes polychlorinated biphenyl (PCB) doserelated depression of choline acetyltransferase (ChAT) activity in hippocampus and basal forebrain of 15-day-old rats, Toxicology 94 (1) (1994) 19-30.

[10] H.Q. Xie, et al., AhR-mediated effects of dioxin on neuronal acetylcholinesterase expression in vitro, Environ. Health Perspect. 121 (5) (2013) 613-618.

[11] H. Hanieh, Toward understanding the role of aryl hydrocarbon receptor in the immune system: current progress and future trends, Biomed. Res. Int. 2014 (2014) 520763.

[12] H. Hanieh, A. Alzahrani, MicroRNA-132 suppresses autoimmune encephalomyelitis by inducing cholinergic anti-inflammation: a new Ahr-based exploration, Eur. J. Immunol. 43 (10) (2013) 2771-2782, 11.

[13] H.Q. Xie, et al., PRiMA directs a restricted localization of tetrameric AChE at synapses, Chem. Biol. Interact 187 (1) (2010) 78-83.

[14] R.C. Choi, et al., Activation of UTP-sensitive P2Y2 receptor induces the expression of cholinergic genes in cultured cortical neurons: a signaling cascade triggered by $\mathrm{Ca}^{2+}$ mobilization and extracellular regulated kinase phosphorylation, Mol. Pharmacol. 84 (1) (2013) 50-61.

[15] A. El Omri, et al., Rosmarinus officinalis polyphenols activate cholinergic activities in PC12 cells through phosphorylation of ERK1/2, J. Ethnopharmacol. 131 (2) (2010) 451-458.

[16] A. El Omri, et al., Luteolin enhances cholinergic activities in PC12 cells through ERK1/2 and PI3K/Akt pathways, Brain Res. 1437 (2012) 16-25.

[17] S.Y. Kim, J.H. Yang, Neurotoxic effects of 2,3,7,8-tetrachlorodibenzo-p-dioxin in cerebellar granule cells, Exp. Mol. Med. 37 (1) (2005) 58-64.

[18] J. Borlak, H.S. Jenke, Cross-talk between aryl hydrocarbon receptor and mitogen-activated protein kinase signaling pathway in liver cancer through craf transcriptional regulation, Mol. Cancer Res. 6 (8) (2008) 1326-1336.

[19] N. Aguilera-Montilla, et al., Aryl hydrocarbon receptor contributes to the MEK/ ERK-dependent maintenance of the immature state of human dendritic cells, Blood 121 (15) (2013) e108-e117.

[20] H. Yu, et al., The aryl hydrocarbon receptor suppresses osteoblast proliferation and differentiation through the activation of the ERK signaling pathway, Toxicol. Appl. Pharmacol. 280 (3) (2014) 502-510.

[21] K.W. Tsim, et al., Transcriptional control of different subunits of AChE in muscles: signals triggered by the motor nerve-derived factors, Chem. Biol. Interact. 175 (1) (2008) 58-63.

[22] N.L. Siow, et al., A cyclic AMP-dependent pathway regulates the expression of acetylcholinesterase during myogenic differentiation of C2C12 cells, J. Biol. Chem. 277 (39) (2002) 36129-36136.

[23] H.Q. Xie, et al., Regulation of a transcript encoding the proline-rich membrane anchor of globular muscle acetylcholinesterase. The suppressive roles of myogenesis and innervating nerves, J. Biol. Chem. 282 (16) (2007) 11765-11775.

[24] R.C. Choi, et al., Calcitonin gene-related peptide induces the expression of acetylcholinesterase-associated collagen ColO in muscle: a distinction in driving two different promoters between fast- and slow-twitch muscle fibers, J. Neurochem. 102 (4) (2007) 1316-1328.

[25] R.C. Choi, et al., Expression of CAMP-responsive element binding proteins (CREBs) in fast-and slow-twitch muscles: A signaling pathway to account for the synaptic expression of collagen-tailed subunit (ColQ) of acetylcholinesterase at the rat neuromuscular junction, Chem. Biol. Interact. 203 (1) (2013) 282-286.

[26] B. Oesch-Bartlomowicz, et al., Aryl hydrocarbon receptor activation by cAMP vs. dioxin: divergent signaling pathways, Proc. Natl. Acad. Sci. U. S. A. 102 (26) (2005) 9218-9223.

[27] H. Takanaga, et al., Inhibitory effect of 2, 3, 7, 8-tetrachlorodibenzo-p-dioxin on cAMP-induced differentiation of rat C6 glial cell line, J. Neurosci. Res. 64 (4) (2001) 402-409.

[28] A. Morales-Hernández, et al., 2,3,7,8-Tetrachlorodibenzo-p-dioxin induces apoptosis by disruption of intracellular calcium homeostasis in human neuronal cell line SHSY5Y, Apoptosis 17 (11) (2012) 1170-1181.

[29] S. Shyamasundar, et al., Epigenetic mechanisms in nanomaterial-induced toxicity, Epigenomics 7 (3) (2015) 395-411.

[30] K.M. Lau, et al., Transcriptional activity of acetylcholinesterase gene is regulated by DNA methylation during C2C12 myogenesis, Brain Res. 1642 (2016) $114-123$.

[31] B.S. Sailaja, et al., Stress-induced epigenetic transcriptional memory of acetylcholinesterase by HDAC4, Proc. Natl. Acad. Sci. 109 (52) (2012) E3687-E3695.

[32] E.A. McClure, et al., Changes in DNA methylation and gene expression during 2,3,7,8-tetrachlorodibenzo-p-dioxin-induced suppression of the lipopolysaccharide-stimulated IgM response in splenocytes, Toxicol. Sci. 120 (2) (2011) 339-348.

[33] L. Lind, et al., Global DNA hypermethylation is associated with high serum levels of persistent organic pollutants in an elderly population, Environ. Int. 59 (2013) 456-461.

[34] A. Cellerino, L. Bally-Cuif, T. Pizzorusso, Editorial for "Regulatory RNAs in the nervous system", Front. Cell. Neurosci. 9 (2015).

[35] G. Shaltiel, et al., Hippocampal microRNA-132 mediates stress-inducible cognitive deficits through its acetylcholinesterase target, Brain Struct. Funct. 218 (1) (2013) 59-72.

[36] G. Hanin, et al., Competing targets of microRNA-608 affect anxiety and hypertension, Hum. Mol. Genet. 23 (17) (2014) 4569-4580.

[37] G. Hanin, H. Soreq, Cholinesterase-targeting microRNAs identified in silico affect specific biological processes, Front. Mol. Neurosci. 4 (2011). 
[38] A. Lesiak, et al., The environmental neurotoxicant PCB 95 promotes synaptogenesis via ryanodine receptor-dependent miR132 upregulation, J. Neurosci. 34 (3) (2014) 717-725.

[39] E. Elyakim, et al., hsa-miR-191 is a candidate oncogene target for hepatocellular carcinoma therapy, Cancer Res. 70 (20) (2010) 8077-8087.

[40] S. Zhang, et al., Aryl hydrocarbon receptor agonists induce microRNA-335 expression and inhibit lung metastasis of estrogen receptor negative breast cancer cells, Mol. Cancer Ther. 11 (1) (2012) 108-118.

41] $\mathrm{H}$. Hanieh, Aryl hydrocarbon receptor-microRNA-212/132 axis in human breast cancer suppresses metastasis by targeting SOX4, Mol. Cancer 14 (2015) 172.

[42] M.W. Gordon, et al., Regulation of p53-targeting microRNAs by polycyclic aromatic hydrocarbons: implications in the etiology of multiple myeloma, Mol. Carcinog. 54 (10) (2015) 1060-1069. 